\title{
Mittag-Leffler-Hyers-Ulam stability of differential equation using Fourier transform
}

\author{
Arusamy Mohanapriya ${ }^{1}$, Choonkil Park ${ }^{2 *}$, Anumanthappa Ganesh ${ }^{3}$ and Vediyappan Govindan ${ }^{4}$
}

${ }^{\text {*Correspondence: }}$

baak@hanyang.ac.kr

${ }^{2}$ Research Institute for Natural

Sciences, Hanyang University, Seoul

04763, Korea

Full list of author information is

available at the end of the article

\section{Springer}

\begin{abstract}
This research paper aims to present the results on the Mittag-Leffler-Hyers-Ulam and Mittag-Leffler-Hyers-Ulam-Rassias stability of linear differential equations of first, second, and $n$th order by the Fourier transform method. Moreover, the stability constant of such equations is obtained. Some examples are given to illustrate the main results.
\end{abstract}

MSC: 58F15; 58F17; 53C35; 39B82; 39B62; 39B52

Keywords: Mittag-Leffler-Hyers-Ulam stability; Fourier transform; Differential equation

\section{Introduction}

In recent years, there has been subject so far-reaching of research in derivative and differential equation because of its performance in numerous branches of pure and applied mathematics. The standards of differential equation have been unlimited and characterize physical models of many phenomena in various fields (see [1]).

As we all know, the main difficulty to find exact solution of such equation is very crucial, and the form of the exact solution (if it exists) is often so arduous that it is not appropriate for numerical calculation. In view of this, it is imperative to discuss approximate solution and ask whether it lies near the exact solution. Mostly, we say that a differential equation is stable in the Hyers-Ulam sense if, for every solution of the differential equation, there exists an approximate solution of the perturbed equation that is close to it.

The history of Hyers-Ulam stability starts from the middle of the nineteenth century. The class of stability was first formulated by Ulam [2] for functional equation which was solved by Hyers [3] for an additive function defined on a Banach space. After this result, the stability concept was investigated and generalized by Rassias [4], which is called HyersUlam-Rassias stability. Further, Alsina and Ger [5] established the Hyers-Ulam stability of differential equations by replacing functional equation. Rezaei and Jung and Rassias [6] investigated the Hyers-Ulam stability of linear differential equation by applying the Laplace transform method. In [7], Algifiary and Jung gave Hyers-Ulam stability of $n$th order linear differential equation with the help of the Laplace transform method.

Using the Hyers-Ulam method, Wu and Baleanu [8] proved the Mittag-Leffler stability of impulsive fractional difference equations; Wu, Baleanu, and Huang [9] proved the

(c) The Author(s) 2020. This article is licensed under a Creative Commons Attribution 4.0 International License, which permits use, sharing, adaptation, distribution and reproduction in any medium or format, as long as you give appropriate credit to the original author(s) and the source, provide a link to the Creative Commons licence, and indicate if changes were made. The images or other third party material in this article are included in the article's Creative Commons licence, unless indicated otherwise in a credit line to the material. If material is not included in the article's Creative Commons licence and your intended use is not permitted by statutory regulation or exceeds the permitted use, you will need to obtain permission directly from the copyright holder. To view a copy of this licence, visit http://creativecommons.org/licenses/by/4.0/. 
Mittag-Leffler stability of linear fractional delay difference equations with impulse, and Wu et al. [10] investigated the Mittag-Leffler stability analysis of fractional discrete-time neural networks via the fixed point technique.

In this paper, we introduce some new concepts concerning the stability of differential equation in the Mittag-Leffler-Hyers-Ulam sense by the Fourier transform method. The Fourier transform and Mittag-Leffler function are effective tools for analytic expression for the solution of linear differential equation of integer or noninteger order. The Mittag-Leffler function $E_{\alpha}\left(z^{\alpha}\right)$ was introduced by Mittag-Leffler [11] in connection with the method of divergent series. The generalization and properties of $E_{\alpha}\left(z^{\alpha}\right)$ were studied and discussed in [12-15]. The Fourier transform is a kind of integral transform, and it was used by Fourier in 1807. It converts differential equation into simple algebraic equation. After solving the algebraic equation, we can find the solution of the original equation by inverse Fourier transform. For more details, see [16, 17].

At present, some remarkable results to Hyers-Ulam-Mittag-Leffler stability of differential equation have been reported in [18-28]. In particular, Kalvandi, Eghbali, and Rassias [18] discussed Mittag-Leffler-Hyers-Ulam stability for the second-order differential equation

$$
y^{\prime \prime}+\alpha y^{\prime}+\beta y=0
$$

and also proved the stability of Lane-Emden equation of second order. Existence and uniqueness of Mittag-Leffler-Ulam stable solution for fractional integro-differential equation with nonlocal initial condition have been proved in [22]. In 2020, Liu et al. studied Hyers-Ulam stability and existence of solutions for fractional differential equation with Mittag-Leffler kernel [20]. To the best of our knowledge, there are few results on MittagLeffler-Hyers-Ulam stability of differential equation by the Fourier transform method.

Motivated by ongoing research on the stability of differential equation, in this paper, we discuss the existence and the Mittag-Leffler-Hyers-Ulam stability of linear homogeneous differential equation

$$
\mathcal{H}^{n}(x)+\sum_{j=0}^{n-1} a_{j} \mathcal{H}^{j}(x)=0, \quad \lim _{|x| \rightarrow \infty} \mathcal{H}(x)=0
$$

with the help of Fourier transform.

The contribution of the paper is outlined as follows: In Sect. 2, some definitions, lemmas, and theorems are introduced. In Sect. 3, the Hyers-Ulam-Mittag-Leffler stability of differential equation of first, second, and nth order is presented. The conclusion and examples are given in Sects. 4 and 5, respectively.

\section{Preliminaries}

In this section, we recall some basic definitions, notations, and theorems for further work. Throughout this paper, let $\mathbb{F}$ be either a real field $\mathbb{R}$ or a complex field $\mathbb{C}$.

Definition 2.1 ([16]) If a function $\mathcal{H}: \mathbb{R} \rightarrow \mathbb{F}$ is piecewise continuous in each finite interval and is absolutely integrable in $\mathbb{R}$, then the Fourier transform associated with $\mathcal{H} \in L^{\prime}(\mathbb{R})$ 
is a mapping $\widehat{\mathcal{H}}(\xi): \mathbb{R} \rightarrow \mathbb{F}$ given by the integral

$$
\widehat{\mathcal{H}}(\xi)=\int_{-\infty}^{\infty} \mathcal{H}(x) e^{i \xi x} d x \quad \forall x \in \mathbb{R} .
$$

Also the inverse Fourier transform associated with $\widehat{\mathcal{H}}(\xi)$ is given by

$$
\mathcal{H}(x)=\frac{1}{2 \pi} \int_{-\infty}^{\infty} \widehat{\mathcal{H}}(\xi) e^{-i \xi x} d \xi
$$

for any $x \in \mathbb{R}$, and the relation $F^{-1} F(\mathcal{H})=\mathcal{H}$ holds true almost everywhere on $\mathbb{R}$.

In the following, we give some properties of the Fourier transform which are closely related to solution process.

Lemma 2.2 $([29,30])$ Let $\mathcal{H} \in L^{\prime}(\mathbb{R}), F(\mathcal{H})(x)=\widehat{\mathcal{H}}(\xi)$, and $\theta(x)$ be the Heaviside step function defined by $\theta(x)=1$ for $x \geq 0$ and $\theta(x)=0$ for $x<0$. Then

1. $\quad F(\mathcal{H}(x \pm a))=e^{ \pm i a} \widehat{\mathcal{H}}(\xi)$;

2. $\quad F\left(e^{-z x} \theta(x)\right)(\xi)=\frac{1}{i \xi+z}$ provided that $\operatorname{Re}(z)>0$;

3. $\quad F\left((-i x)^{n}(\mathcal{H}(x))(\xi)=F^{n}(\xi)\right.$;

4. $\quad F\left((\mathcal{H})^{n}(x)\right)(\xi)=(-i \xi)^{n} \widehat{\mathcal{H}}(\xi)$.

The convolution of two functions $\mathcal{H}_{1}(x)$ and $\mathcal{H}_{2}(x)$ is defined as

$$
\mathcal{H}_{1}(x) * \mathcal{H}_{2}(x)=\int_{-\infty}^{\infty} \mathcal{H}_{1}(\mu) \mathcal{H}_{2}(x-\mu) d \mu
$$

We have the following theorem.

Theorem 2.3 ([30]) Let $\mathcal{H}_{1}, \mathcal{H}_{2} \in L^{1}(\mathbb{R})$. Then

1. $\quad F\left(\mathcal{H}_{1} * \mathcal{H}_{2}\right)=F\left(\mathcal{H}_{1}\right) F\left(\mathcal{H}_{2}\right)$;

2. $F^{-1}\left(\mathcal{H}_{1} \mathcal{H}_{2}\right)=F^{-1}\left(\mathcal{H}_{1}\right) * F^{-1}\left(\mathcal{H}_{2}\right)$.

Notice that if $\theta(x)$ is the Heaviside step function, then

$$
(h * \theta)(x)=(\theta * h)(x)=\int_{\infty}^{\infty} \theta(x-\mu) h(\mu) d \mu=\int_{0}^{\infty} h(\mu) d \mu .
$$

Definition 2.4 ([11]) The Mittag-Leffler function of one parameter is defined as

$$
E_{\alpha}(z)=\sum_{k=0}^{\infty} \frac{1}{\Gamma \alpha k+1} z^{k}
$$

where $\operatorname{Re}(\alpha)>0$ and $z, \alpha \in \mathbb{C}$.

Definition 2.5 The two-parameter Mittag-Leffler function is denoted by $E_{\alpha, \beta}(z)$ and is defined as

$$
E_{\alpha, \beta}(z)=\sum_{k=0}^{\infty} \frac{1}{\Gamma \alpha k+\beta} z^{k} .
$$


When $\alpha=\beta=1$, the above equation becomes

$$
E_{1,1}(z)=\sum_{k=0}^{\infty} \frac{1}{\Gamma k+1} z^{k}
$$

Theorem 2.6 For any $x, \alpha \in \mathbb{C}$ with $\operatorname{Re}(\alpha)>0$, the Fourier transform of Mittag-Leffler function is

$$
F\left(E_{\alpha}(x)\right)=\sum_{k=0}^{\infty} \frac{k !}{\Gamma \alpha k+1} i^{-k-1} \xi^{-(k+1)}
$$

Proof By Mittag-Leffler function of one parameter for $x \in \mathbb{R}$, we get

$$
E_{\alpha}(x)=\sum_{k=0}^{\infty} \frac{1}{\Gamma \alpha k+1} x^{k}
$$

Taking Fourier transform of (2.3), we have

$$
F\left(E_{\alpha}(x)\right)=\int_{-\infty}^{\infty} E_{\alpha}(x) e^{i \xi x} d x=\sum_{k=0}^{\infty} \frac{1}{\Gamma \alpha k+1} \int_{-\infty}^{\infty} x^{k} e^{i \xi x} d x
$$

Letting $i \xi x=-z, i \xi d x=-d z$, we get

$$
\begin{aligned}
F\left(E_{\alpha}(x)\right) & =\sum_{k=0}^{\infty} \frac{1}{\Gamma \alpha k+1} \int_{0}^{\infty}\left(\frac{z}{i \xi}\right)^{k} e^{-z} \frac{d z}{i \xi} \\
& =\sum_{k=0}^{\infty} \frac{1}{\Gamma \alpha k+1} i^{-k-1} \xi^{-(k+1)} \int_{0}^{\infty} z^{k} e^{-z} d z
\end{aligned}
$$

Since $\int_{0}^{\infty} z^{k} e^{-z} d z=\Gamma(k+1)=k$ !, we obtain

$$
F\left(E_{\alpha}(x)\right)=\sum_{k=0}^{\infty} \frac{k !}{\Gamma \alpha k+1} i^{-k-1} \xi^{-(k+1)} .
$$

This completes the proof.

\section{Main results}

In this section, we study the existence and stability for differential equation (1.1). Moreover, we derive the stability constant for Eq. (1.1).

\subsection{Mittag-Leffler-Hyers-Ulam stability of linear differential equation of first order}

In this subsection, by means of Fourier transform and convolution principle, we establish the stability of the homogeneous first-order differential equation

$$
\mathcal{H}^{\prime}(x)+a \mathcal{H}(x)=0, \quad \lim _{|x| \rightarrow \infty} \mathcal{H}(x)=0
$$

where $\mathcal{H}(x)$ is a continuously differentiable function and $a$ is a constant. 
Definition 3.1 We say that linear differential equation (3.1) is said to have Mittag-LefflerHyers-Ulam stability if there exists a constant $K>0$ with the following: for every $\epsilon>0$ and a continuously differentiable function $\mathcal{H}(x)$ satisfying the inequality

$$
\left|\mathcal{H}^{\prime}(x)+a \mathcal{H}(x)\right| \leq \epsilon E_{\alpha}\left(x^{\alpha}\right)
$$

there exists some $\mathcal{H}_{o}(x)$ satisfying differential equation (3.1) such that

$$
\left|\mathcal{H}(x)-\mathcal{H}_{o}(x)\right| \leq K \epsilon E_{\alpha}\left(x^{\alpha}\right),
$$

where $K$ is a Mittag-Leffler-Hyers-Ulam stability constant.

Remark 1 If $\epsilon$ and $K \epsilon$ are replaced by continuous functions $\phi(x)$ and $\Phi(x)$ in the above definition, then we say that Eq. (3.1) has Hyers-Ulam-Mittag-Leffler-Rassias stability.

Theorem 3.2 Let a be a scalar in $\mathbb{F}$. Assume that, for every $\epsilon>0$, there exists $K>0$ such that $\mathcal{H}(x) \in L^{\prime}(\mathbb{R})$ satisfying the differential inequality

$$
\left|\mathcal{H}^{\prime}(x)+a \mathcal{H}(x)\right| \leq \epsilon E_{\alpha}\left(x^{\alpha}\right)
$$

for all $x \in \mathbb{R}$. Then there exists a solution $\mathcal{H}(x) \in L^{\prime}(\mathbb{R})$ of differential equation (3.1) such that

$$
\left|\mathcal{H}(x)-\mathcal{H}_{o}(x)\right| \leq K \epsilon E_{\alpha}\left(x^{\alpha}\right)
$$

for all $x \in \mathbb{R}$.

Proof Assume that a continuously differentiable function $\mathcal{H}(x)$ satisfies inequality (3.3). First, let us find the classic solution of (3.1). Apply the derivative of Fourier transform

$$
F\left(\mathcal{H}^{\prime}(x)\right)=(-i \xi) \widehat{\mathcal{H}}(\xi)
$$

with respect to the variable $x$. Here $\widehat{\mathcal{H}}(\xi)$ is the Fourier transform of $\mathcal{H}(x)$. Then (3.1) reduces to

$$
(-i \xi+a) \widehat{\mathcal{H}}(\xi)=0
$$

Thus the solution of transformed equation (3.4) is

$$
\mathcal{H}_{o}(x)=C e^{a x}, \quad \forall x \in \mathbb{R}
$$

where $C$ is a constant. Introduce a function $\eta:(-\infty, \infty) \rightarrow \mathcal{F}$ such that

$$
\eta(x)=\mathcal{H}^{\prime}(x)+a \mathcal{H}(x) .
$$

Suppose that $|\eta(x)| \leq \epsilon E_{\alpha}\left(x^{\alpha}\right)$. By taking the Fourier transform of (3.6), it is transformed into

$$
(-i \xi+a) \widehat{\mathcal{H}}(\xi)=\widehat{\mathcal{H}}(\xi)
$$


The method of variation of constant gives the unique solution of (3.7), which is

$$
\begin{aligned}
\mathcal{H}(x) & =C e^{a x}+F^{-1}\left(\widehat{\eta}(x) \frac{1}{i \xi-a}\right) \\
& =C e^{a x}+F^{-1}(\widehat{\eta}(x)) * F^{-1}\left(\frac{1}{i \xi-a}\right) .
\end{aligned}
$$

Applying the property of Fourier transform and the formula of convolution, we obtain

$$
\mathcal{H}(x)=C e^{a x}+\eta(x) * e^{a x} \theta(x)=C e^{a x}+\int_{-\infty}^{\infty} \eta(\mu) e^{a(x-\mu)} d \mu .
$$

It follows from (3.5) and (3.8) that

$$
\begin{aligned}
\left|\mathcal{H}(x)-\mathcal{H}_{o}(x)\right| & \leq\left|\int_{-\infty}^{\infty} \eta(\mu) e^{a(x-\mu)} \theta(x-\mu) d \mu\right| \\
& \leq \epsilon \sum_{k=0}^{\infty} \frac{1}{\Gamma(\alpha k+1)} \int_{0}^{x} \mu^{\alpha k} d \mu \\
& \leq \epsilon \sum_{k=0}^{\infty} \frac{1}{\Gamma(\alpha k+1)} \frac{x^{\alpha k+1}}{\alpha k+1},
\end{aligned}
$$

and so

$$
\left|\mathcal{H}(x)-\mathcal{H}_{o}(x)\right| \leq \epsilon K E_{\alpha, 2}\left(x^{\alpha}\right)
$$

for all $x>0$. Clearly, this implies that the homogeneous linear differential equation (3.1) has Mittag-Leffler-Hyers-Ulam stability.

Similarly, we can explore Mittag-Leffler-Hyers-Ulam-Rassias stability of differential equation (3.1).

Corollary 1 For every continuously differential function $\mathcal{H}(x) \in L^{\prime}(\mathbb{R})$ satisfying the differential inequality

$$
\left|\mathcal{H}^{\prime}(x)+a \mathcal{H}(x)\right| \leq \phi(x) E_{\alpha}\left(x^{\alpha}\right) \quad \forall x \in \mathbb{R}
$$

there exists a solution $\mathcal{H}_{o}(x) \in L^{\prime}(\mathbb{R})$ of differential equation (3.1) such that

$$
\left|\mathcal{H}(x)-\mathcal{H}_{o}(x)\right| \leq K \Phi(x) E_{\alpha}\left(x^{\alpha}\right) \quad \forall x \in \mathbb{R}
$$

\subsection{Mittag-Leffler-Hyers-Ulam stability of linear differential equation of second order}

In this subsection, we are going to verify that the approximate solution is near the exact solution for the linear differential equation of second order

$$
\mathcal{H}^{\prime \prime}(x)+a \mathcal{H}^{\prime}(x)+b \mathcal{H}(x)=0, \quad \lim _{|x| \rightarrow \infty} \mathcal{H}(x)=0
$$

with the help of the Fourier transform method. 
Definition 3.3 The linear differential equation (3.10) is said to have Mittag-LefflerHyers-Ulam stability if there exists a constant $K>0$ with the following property: for every $\epsilon>0$ and a continuously differentiable function $\mathcal{H}(x) \in L^{\prime}(\mathbb{R})$ satisfying the inequality

$$
\left|\mathcal{H}^{\prime \prime}(x)+a \mathcal{H}^{\prime}(x)+b \mathcal{H}(x)\right| \leq \epsilon E_{\alpha}\left(x^{\alpha}\right)
$$

where $E_{\alpha}$ is a Mittag-Leffler function, there exists some $\mathcal{H}_{o}(x) \in L^{\prime}(\mathbb{R})$ satisfying differential equation (3.10) such that

$$
\left|\mathcal{H}(x)-\mathcal{H}_{o}(x)\right| \leq K \epsilon E_{\alpha}\left(x^{\alpha}\right) .
$$

Theorem 3.4. Assume that the characteristic equation of (3.10) has two different positive roots. If, for every $\epsilon>0, \mathcal{H}(x) \in L^{\prime}(\mathbb{R})$ satisfies the inequality

$$
\left|\mathcal{H}^{\prime \prime}(x)+a \mathcal{H}^{\prime}(x)+b \mathcal{H}(x)\right| \leq \epsilon E_{\alpha}\left(x^{\alpha}\right),
$$

then there exist some $\mathcal{H}_{o}(x) \in L^{\prime}(\mathbb{R})$ and $K>0$ satisfying (3.10) such that

$$
\left|\mathcal{H}(x)-\mathcal{H}_{0}(x)\right| \leq K \epsilon E_{\alpha}\left(x^{\alpha}\right),
$$

that is, Eq. (3.10) has Mittag-Leffler-Hyers-Ulam stability.

Proof Let $\epsilon>0$ and $\mathcal{H}(x) \in L^{\prime}(\mathbb{R})$ such that

$$
\left|\mathcal{H}(x)-\mathcal{H}_{0}(x)\right| \leq K \epsilon E_{\alpha}\left(x^{\alpha}\right) .
$$

First, we will compute the classical solution of (3.10). Apply the Fourier transform with respect to variable $x$ defined by (2.1) to (3.10). By

$$
F\left(\mathcal{H}^{\prime}(x)\right)=-i \xi \widehat{\mathcal{H}}(\xi), \quad F\left(\mathcal{H}^{\prime \prime}(x)\right)=(-i \xi)^{2} \widehat{\mathcal{H}}(\xi)
$$

where $\widehat{\mathcal{H}}(\xi)$ is the Fourier transform of $\mathcal{H}(x),(3.10)$ reduces to

$$
\left((-i \xi)^{2}+(-i \xi) a+b\right) \widehat{\mathcal{H}}(\xi)=0 .
$$

Let $\mathcal{M}_{1}$ and $\mathcal{M}_{2}$ be distinct roots of the characteristic equation of (3.12)

$$
\mathcal{M}^{2}+a \mathcal{M}+b=0 \text {. }
$$

Since $a, b$ are constant in $\mathbb{F}$ such that

$$
\mathcal{M}_{1}+\mathcal{M}_{2}=-a, \quad \mathcal{M}_{1} \mathcal{M}_{2}=b,
$$

we have $(-i \xi)^{2}+(-i \xi) a+b=\left(i \xi-\mathcal{M}_{1}\right)\left(i \xi-\mathcal{M}_{2}\right)$.

Thus the solution of transformed equation (3.12) is

$$
\mathcal{H}_{o}(x)=C_{1} e^{-\mathcal{M}_{1}(x)}+C_{2} e^{-\mathcal{M}_{2}(x)},
$$


where $C_{1}$ and $C_{2}$ are constant. Now, we introduce the function

$$
\eta(x)=\mathcal{H}^{\prime \prime}(x)+a \mathcal{H}^{\prime}(x)+b \mathcal{H}(x) .
$$

Next, we will show Mittag-Leffler-Hyers-Ulam stability of (3.10). By taking the Fourier transform of (3.14), it is transformed into

$$
(-i \xi)^{2} \widehat{\mathcal{H}}(\xi)+a(-i \xi) \widehat{\mathcal{H}}(\xi)+b \widehat{\mathcal{H}}(\xi)=\widehat{\eta}(\xi)
$$

The method of variation of constant gives the unique solution of (3.15), which is

$$
\begin{aligned}
\mathcal{H}(x) & =C_{1} e^{-\mathcal{M}_{1}(x)}+C_{2} e^{-\mathcal{M}_{2}(x)}+F^{-1}\left(\widehat{\eta}(\xi) \frac{1}{\left(i \xi-\mathcal{M}_{1}\right)\left(i \xi-\mathcal{M}_{2}\right)}\right) \\
& =C_{1} e^{-\mathcal{M}_{1}(x)}+C_{2} e^{-\mathcal{M}_{2}(x)}+F^{-1}(\widehat{\eta}(\xi)) * F^{-1}\left(\frac{1}{\left(i \xi-\mathcal{M}_{1}\right)\left(i \xi-\mathcal{M}_{2}\right)}\right) .
\end{aligned}
$$

Set $\widehat{Q}(\xi)=\frac{1}{\left(i \xi-\mathcal{M}_{1}\right)\left(i \xi-\mathcal{M}_{2}\right)}=\frac{1}{\mathcal{M}_{2}-\mathcal{M}_{1}}\left(\frac{1}{\left(i \xi-\mathcal{M}_{1}\right)\left(i \xi-\mathcal{M}_{2}\right)}\right)$.

By the inverse Fourier transform, we get

$$
F^{-1}(\widehat{Q}(\xi))=q(x)=\frac{1}{\mathcal{M}_{2}-\mathcal{M}_{1}}\left(F^{-1}\left(\frac{1}{\left(i \xi-\mathcal{M}_{1}\right)}\right)-F^{-1}\left(\frac{1}{\left(i \xi-\mathcal{M}_{2}\right)}\right)\right) .
$$

By taking account of the property of Fourier transform, we get

$$
q(x)=\frac{1}{\mathcal{M}_{2}-\mathcal{M}_{1}}\left(e^{\mathcal{M}_{1} x} \theta(x)-e^{\mathcal{M}_{2} x} \theta(x)\right)
$$

where $\theta(x)$ is a Heaviside step function. (3.16) becomes

$$
\mathcal{H}(x)=C_{1} e^{-\mathcal{M}_{1}(x)}+C_{2} e^{-\mathcal{M}_{2}(x)}+\eta(x) * q(x) .
$$

Applying the formula of convolution, we obtain

$$
\mathcal{H}(x)=C_{1} e^{-\mathcal{M}_{1}(x)}+C_{2} e^{-\mathcal{M}_{2}(x)}+\int_{-\infty}^{\infty} \eta(\mu) q(x-\mu) d \mu .
$$

It follows from (3.13) and (3.17) that

$$
\begin{aligned}
\left|\mathcal{H}(x)-\mathcal{H}_{o}(x)\right| & =|\eta(\mu) q(x-\mu) d \mu| \\
& \leq \frac{\epsilon}{\mathcal{M}_{2}-\mathcal{M}_{1}} \sum_{k=0}^{\infty} \frac{1}{\Gamma(\alpha k+1)} \int_{-\infty}^{\infty} \mu^{\alpha k}\left(e^{\mathcal{M}_{1}(x-\mu)}-e^{\mathcal{M}_{2}(x-\mu)}\right) \theta(\mu) d \mu \\
& \leq \frac{\epsilon}{\mathcal{M}_{2}-\mathcal{M}_{1}} \sum_{k=0}^{\infty} \frac{1}{\Gamma(\alpha k+1)} \int_{0}^{\infty} \mu^{\alpha k}\left(e^{\mathcal{M}_{1}(x-\mu)}-e^{\mathcal{M}_{2}(x-\mu)}\right) d \mu
\end{aligned}
$$


for all $x>0$, and so we get

$$
\begin{aligned}
\left|\mathcal{H}(x)-\mathcal{H}_{o}(x)\right| & \leq \frac{\epsilon}{\mathcal{M}_{2}-\mathcal{M}_{1}} \sum_{k=0}^{\infty} \frac{1}{\Gamma(\alpha k+1)} \int_{0}^{x} \mu^{\alpha k}\left(e^{\mathcal{M}_{1}(x-\mu)}-e^{\mathcal{M}_{2}(x-\mu)}\right) d \mu \\
& \leq \frac{\epsilon}{\mathcal{M}_{2}-\mathcal{M}_{1}} \sum_{k=0}^{\infty} \frac{1}{\Gamma(\alpha k+1)} \int_{0}^{x} \mu^{\alpha k} d \mu \\
& =\frac{\epsilon}{\mathcal{M}_{2}-\mathcal{M}_{1}} \sum_{k=0}^{\infty} \frac{1}{\Gamma(\alpha k+1)} \frac{x^{\alpha k+1}}{\alpha k+1}
\end{aligned}
$$

and so

$$
\left|\mathcal{H}(x)-\mathcal{H}_{o}(x)\right| \leq K \epsilon E_{\alpha, 2}\left(x^{\alpha}\right)
$$

This completes the proof of the theorem.

Similarly, we can explore that Mittag-Leffler-Hyers-Ulam-Rassias stability of differential equation (3.10).

Corollary 2 Let a be a scalar in $\mathbb{F}$ and $\mathcal{H}(x) \in L^{\prime}(\mathbb{R})$. Assume that there exists a constant $K>0$ such that $\mathcal{H}(x) \in L^{\prime}(\mathbb{R})$ satisfies the differential inequality

$$
\left|\mathcal{H}^{\prime \prime}(x)+a \mathcal{H}^{\prime}(x)+b \mathcal{H}(x)\right| \leq \phi(x) E_{\alpha}\left(x^{\alpha}\right)
$$

for all $x \in \mathbb{R}$. Then there exists a solution $\mathcal{H}_{o}(x) \in L^{\prime}(\mathbb{R})$ of differential equation (3.10) such that

$$
\left|\mathcal{H}(x)-\mathcal{H}_{o}(x)\right| \leq K \Phi(x) E_{\alpha}\left(x^{\alpha}\right)
$$

for all $x \in \mathbb{R}$, i.e., Eq. (3.10) has Mittag-Leffler-Hyers-Ulam-Rassias stability.

\subsection{Mittag-Leffler-Hyers-Ulam stability of linear differential equation of $n$th order}

Now, we give the proof of Mittag-Leffler-Hyers-Ulam stability of the linear differential equation of $n$th order

$$
\mathcal{H}^{n}(x)+\sum_{j=0}^{n-1} a_{j} \mathcal{H}^{j}(x)=0, \quad \lim _{|x| \rightarrow \infty} \mathcal{H}(x)=0 .
$$

Definition 3.5 The linear differential equation (3.18) is said to have Mittag-LefflerHyers-Ulam stability if there exists a constant $K>0$ with the following property: for every $\epsilon>0$ and a continuously differentiable function $\mathcal{H}(x) \in L^{\prime}(\mathbb{R})$ satisfying the inequality

$$
\left|\mathcal{H}^{n}(x)+a_{n-1} \mathcal{H}^{n-1}(x)+\cdots+a_{1} \mathcal{H}^{1}(x)+a_{0} \mathcal{H}(x)\right| \leq \epsilon E_{\alpha}\left(x^{\alpha}\right),
$$

there exists some $\mathcal{H}_{o}(x)$ satisfying differential equation (1.1) such that

$$
\left|\mathcal{H}(x)-\mathcal{H}_{o}(x)\right| \leq K \epsilon E_{\alpha}\left(x^{\alpha}\right) .
$$


Theorem 3.6 Let $a_{i} \in \mathbb{F}$. Assume that the characteristic equation of (3.18) has $n$ distinct positive roots. If, for any $\epsilon>0, \mathcal{H} \in L^{\prime}(\mathbb{R})$ satisfies the differential inequality

$$
\left|\mathcal{H}^{n}(x)+a_{n-1} \mathcal{H}^{n-1}(x)+\cdots+a_{1} \mathcal{H}^{1}(x)+a_{0} \mathcal{H}(x)\right| \leq \epsilon E_{\alpha}\left(x^{\alpha}\right)
$$

for all $x>0$, then there exists a solution $\mathcal{H}_{o} \in L^{\prime}(\mathbb{R})$ of differential equation (3.18) such that

$$
\left|\mathcal{H}(x)-\mathcal{H}_{o}(x)\right| \leq K \epsilon E_{\alpha}\left(x^{\alpha}\right)
$$

for all $x \in \mathbb{R}$.

Proof Let $\epsilon>0$ and $\mathcal{H}(x) \in L^{\prime}(\mathbb{R})$ such that

$$
\left|\mathcal{H}^{n}(x)+a_{n-1} \mathcal{H}^{n-1}(x)+\cdots+a_{1} \mathcal{H}^{1}(x)+a_{0} \mathcal{H}(x)\right| \leq \epsilon E_{\alpha}\left(x^{\alpha}\right) .
$$

First, we will compute the classical solution of (3.18). By applying the Fourier transform with respect to variable $x$ by using

$$
F\left(\mathcal{H}^{n}(x)\right)=(-i \xi)^{n} \widehat{\mathcal{H}}(\xi)
$$

where $\widehat{\mathcal{H}}(\xi)$ is the Fourier transform of $\mathcal{H}(x),(3.18)$ reduces to

$$
\left((-i \xi)^{n}+a_{n-1}(-i \xi)^{n-1}+\cdots+a_{1}(-i \xi)+a_{0}\right) \widehat{\mathcal{H}}(\xi)=0
$$

Let $\mathcal{M}_{1}, \mathcal{M}_{2}, \ldots, \mathcal{M}_{n}$ be distinct roots of the characteristic equation

$$
\mathcal{M}^{n}+a_{n-1} \mathcal{M}^{n-1}+\cdots+a_{1} \mathcal{M}+a_{0}=0
$$

Since $a_{i}$ are constant in $\mathbb{F}$ such that

$$
\begin{aligned}
& \mathcal{M}_{1}+\mathcal{M}_{2}+\cdots+\mathcal{M}_{n}=-a_{n-1} \\
& \mathcal{M}_{1} \mathcal{M}_{2}+\mathcal{M}_{2} \mathcal{M}_{3}+\cdots+\mathcal{M}_{n-1} \mathcal{M}_{n}=a_{n-2}, \\
& \mathcal{M}_{1} \mathcal{M}_{2} \mathcal{M}_{3}+\mathcal{M}_{2} \mathcal{M}_{3} \mathcal{M}_{4}+\cdots+\mathcal{M}_{n-2} \mathcal{M}_{n-1} \mathcal{M}_{n}=-a_{n-3}, \\
& \vdots \\
& \mathcal{M}_{1} \mathcal{M}_{2} \cdots \mathcal{M}_{n}=a_{0}
\end{aligned}
$$

we have

$$
\left((-i \xi)^{n}+a_{n-1}(-i \xi)^{n-1}+\cdots+a_{1}(-i \xi)+a_{0}\right)=\left(i \xi-\mathcal{M}_{1}\right)\left(i \xi-\mathcal{M}_{2}\right) \cdots\left(i \xi-\mathcal{M}_{n}\right)
$$

Thus the solution of transformed equation (3.19) is

$$
\mathcal{H}_{o}(x)=C_{1} e^{-\mathcal{M}_{1}(x)}+C_{2} e^{-\mathcal{M}_{2}(x)}+\cdots+C_{n} e^{-\mathcal{M}_{n}(x)}=\sum_{m=1}^{n} C_{m} e^{-\mathcal{M}_{m}(x)},
$$


where $C_{1}, C_{2}, \ldots, C_{n}$ are constant. Now we introduce the function

$$
\eta(x)=\mathcal{H}^{n}(x)+a_{n-1} \mathcal{H}^{n-1}(x)+\cdots+a_{1} \mathcal{H}^{1}(x)+a_{0} \mathcal{H}(x) .
$$

Next, we will show the Mittag-Leffler-Hyers-Ulam stability of (3.18). By taking the Fourier transform of (3.21), it is transformed into

$$
\left((-i \xi)^{n}+a_{n-1}(-i \xi)^{n-1}+\cdots+a_{1}(-i \xi)+a_{0}\right) \widehat{\mathcal{H}}(\xi)=\widehat{\eta}(\xi)
$$

The method of variation of constant gives the unique solution of (3.22), which is

$$
\begin{aligned}
\mathcal{H}(x) & =\sum_{m=1}^{n} C_{m} e^{-\mathcal{M}_{m}(x)}+F^{-1}\left(\widehat{\eta}(\xi) \frac{1}{\left(i \xi-\mathcal{M}_{1}\right)\left(i \xi-\mathcal{M}_{2}\right) \cdots\left(i \xi-\mathcal{M}_{n}\right)}\right) \\
& =\sum_{m=1}^{n} C_{m} e^{-\mathcal{M}_{m}(x)}+F^{-1}(\widehat{\eta}(\xi)) * F^{-1}\left(\frac{1}{\left(i \xi-\mathcal{M}_{1}\right)\left(i \xi-\mathcal{M}_{2}\right) \cdot\left(i \xi-\mathcal{M}_{n}\right)}\right) .
\end{aligned}
$$

Set

$$
\begin{aligned}
\widehat{Q}(\xi)= & \frac{1}{\left(i \xi-\mathcal{M}_{1}\right)\left(i \xi-\mathcal{M}_{2}\right) \cdots\left(i \xi-\mathcal{M}_{n}\right)} \\
= & \frac{1}{\mathcal{M}_{n}-\mathcal{M}_{n-1}-\cdots-\mathcal{M}_{1}} \\
& \times\left(\left(\frac{1}{\left(i \xi-\mathcal{M}_{1}\right)}\right)-\left(\frac{1}{\left(i \xi-\mathcal{M}_{2}\right)}\right)-\cdots-\left(\frac{1}{\left(i \xi-\mathcal{M}_{n}\right)}\right)\right) .
\end{aligned}
$$

By the inverse Fourier transform, we get

$$
\begin{aligned}
F^{-1}(\widehat{Q}(\xi))= & \frac{1}{\mathcal{M}_{n}-\mathcal{M}_{n-1}-\cdots-\mathcal{M}_{1}} \\
& \times\left(F^{-1}\left(\frac{1}{\left(i \xi-\mathcal{M}_{1}\right)}\right)-F^{-1}\left(\frac{1}{\left(i \xi-\mathcal{M}_{2}\right)}\right)-\cdots-F^{-1}\left(\frac{1}{\left(i \xi-\mathcal{M}_{n}\right)}\right)\right) .
\end{aligned}
$$

By taking account of the property of Fourier transform, we get

$$
q(x)=\frac{1}{\mathcal{M}_{n}-\mathcal{M}_{n-1}-\cdots-\mathcal{M}_{1}}\left(e^{\mathcal{M}_{1} x}-e^{\mathcal{M}_{2} x} \cdots \cdots e^{\mathcal{M}_{n} x}\right) \theta(x),
$$

where $\theta(x)$ is a Heaviside step function. So (3.23) becomes

$$
\mathcal{H}(x)=\sum_{m=1}^{n} C_{m} e^{-\mathcal{M}_{m}(x)}+\eta(x) * q(x) .
$$

By applying the formula of convolution, we obtain

$$
\mathcal{H}(x)=\sum_{m=0}^{n} C_{m} e^{-\mathcal{M}_{m}(x)}+\int_{-\infty}^{\infty} \eta(\mu) q(x-\mu) d \mu
$$


It follows from (3.20) and (3.24) that

$$
\begin{aligned}
& \left|\mathcal{H}(x)-\mathcal{H}_{o}(x)\right|=|\eta(\mu) q(x-\mu) d \mu| \\
& \leq \frac{\epsilon}{\mathcal{M}_{n}-\mathcal{M}_{n-1}-\cdots-\mathcal{M}_{1}} \sum_{k=0}^{\infty} \frac{1}{\Gamma(\alpha k+1)} \\
& \times \int_{-\infty}^{\infty} \mu^{\alpha k}\left(e^{\mathcal{M}_{1}(x-\mu)}-e^{\mathcal{M}_{2}(x-\mu)-\cdots-e^{\mathcal{M}_{n}(x-\mu)}}\right) \theta(x-\mu) d \mu \\
& \leq \frac{\epsilon}{\mathcal{M}_{n}-\mathcal{M}_{n-1}-\cdots-\mathcal{M}_{1}} \sum_{k=0}^{\infty} \frac{1}{\Gamma(\alpha k+1)} \\
& \times \int_{0}^{\infty} \mu^{\alpha k}\left(e^{\mathcal{M}_{1}(x-\mu)}-e^{\mathcal{M}_{2}(x-\mu)}-\cdots-e^{\mathcal{M}_{n}(x-\mu)}\right) d \mu \\
& \leq \frac{\epsilon}{\mathcal{M}_{n}-\mathcal{M}_{n-1}-\cdots-\mathcal{M}_{1}} \sum_{k=0}^{\infty} \frac{1}{\Gamma(\alpha k+1)} \\
& \times \int_{0}^{x} \mu^{\alpha k}\left(e^{\mathcal{M}_{1}(x-\mu)}-e^{\mathcal{M}_{2}(x-\mu)}-\cdots-e^{\mathcal{M}_{n}(x-\mu)}\right) d \mu \\
& \leq \frac{\epsilon}{\mathcal{M}_{n}-\mathcal{M}_{n-1}-\cdots-\mathcal{M}_{1}} \sum_{k=0}^{\infty} \frac{1}{\Gamma(\alpha k+1)} \int_{0}^{x} \mu^{\alpha k} d \mu \\
& =\frac{\epsilon}{\mathcal{M}_{n}-\mathcal{M}_{n-1}-\cdots-\mathcal{M}_{1}} \sum_{k=0}^{\infty} \frac{1}{\Gamma(\alpha k+1)} \frac{x^{\alpha k+1}}{\alpha k+1},
\end{aligned}
$$

and so

$$
\left|\mathcal{H}(x)-\mathcal{H}_{o}(x)\right| \leq K \epsilon E_{\alpha, 2}\left(x^{\alpha}\right)
$$

Hence differential equation (3.18) has Mittag-Leffler-Hyers-Ulam stability.

Similarly, we can prove the Mittag-Leffler-Hyers-Ulam-Rassias stability of Eq. (3.18).

Corollary 3 Assume that the characteristic equation of (3.18) has ' $n$ ' different positive roots. If, for every $\epsilon>0, \mathcal{H}(x) \in L^{\prime}(\mathbb{R})$ satisfies the inequality

$$
\left|\mathcal{H}^{n}(x)+a_{n-1} \mathcal{H}^{n-1}(x)+\cdots+a_{1} \mathcal{H}^{1}(x)+a_{0} \mathcal{H}(x)\right| \leq \phi(x) E_{\alpha}\left(x^{\alpha}\right),
$$

then there exist some $\mathcal{H}_{o}(x) \in L^{\prime}(\mathbb{R})$ and $K>0$ satisfying (3.18) such that

$$
\left|\mathcal{H}(x)-\mathcal{H}_{0}(x)\right| \leq K \Phi(x) E_{\alpha}\left(x^{\alpha}\right)
$$

\section{Numerical examples}

Example 4.1 Consider the following differential equation:

$$
\mathcal{H}^{\prime}(x)+\frac{1}{\sqrt{(1+\exp (7))}} \mathcal{H}(x)=0, \quad \lim _{|x| \rightarrow \infty} \mathcal{H}(x)=0
$$


Figure 1 The solution of Eq. (4.1)

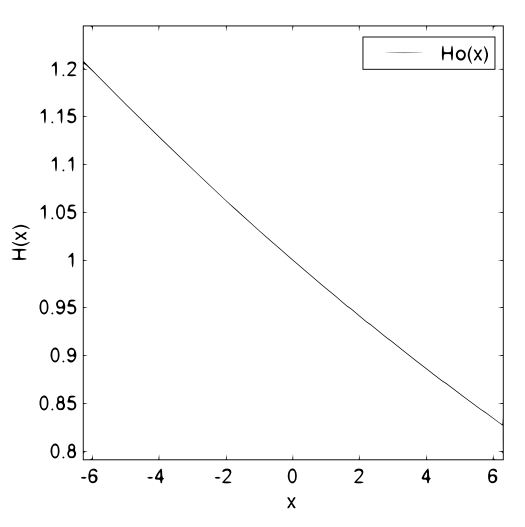

and the inequality

$$
\left|\mathcal{H}^{\prime}(x)+\frac{1}{\sqrt{(1+\exp (7))}} \mathcal{H}(x)\right| \leq \epsilon E_{1}\left(x^{1}\right) \quad \forall x \in \mathbb{R}
$$

where $\mathcal{H} \in L^{\prime}(\mathbb{R})$.

Comparing with (3.1) and (3.3), we have, for $\alpha=1, a=\frac{1}{\sqrt{(1+\exp (7))}}$.

The solution of Eq. (4.1) is computed and depicted in Fig. 1.

By Theorem 3.2, problem (4.1) has a solution and is Hyers-Ulam-Mittag-Leffler stable with

$$
\begin{aligned}
\left|\mathcal{H}(x)-\mathcal{H}_{0}(x)\right| & \leq\left|\int_{0}^{\infty} \eta(\mu) \frac{1}{\sqrt{(1+\exp (7))}} d \mu\right| \\
& \leq K \epsilon E_{1,2}\left(x^{1}\right) .
\end{aligned}
$$

Example 4.2 Consider the following differential equation:

$$
\mathcal{H}^{\prime \prime}(x)+4 i \mathcal{H}(x)=0, \quad \lim _{|x| \rightarrow \infty} \mathcal{H}(x)=0
$$

and the inequality

$$
\left|\mathcal{H}^{\prime \prime}(x)+4 i \mathcal{H}(x)\right| \leq \epsilon E_{2}\left(x^{2}\right) \quad \forall x \in \mathbb{R}
$$

where $\mathcal{H} \in L^{\prime}(\mathbb{R})$.

Comparing with (3.10) and (3.11), we have, for $\alpha=2, a=0$ and $b=4 i$.

Using MATLAB, the solution of Eq. (4.2) is computed and depicted in Fig. 2.

By Theorem 3.4, problem (4.2) has a solution and is Hyers-Ulam-Mittag-Leffler stable with

$$
\begin{aligned}
\left|\mathcal{H}(x)-\mathcal{H}_{0}(x)\right| & \leq \frac{\epsilon}{2 \sqrt{2}(1-i)} \sum_{k=0}^{\infty} \frac{1}{\Gamma(2 k+1)} \int_{0}^{x} \mu^{2 k} d \mu \\
& \leq K \epsilon E_{2,2}\left(x^{2}\right) \quad \forall x \in \mathbb{R} .
\end{aligned}
$$


Figure 2 The solution of Eq. (4.2)

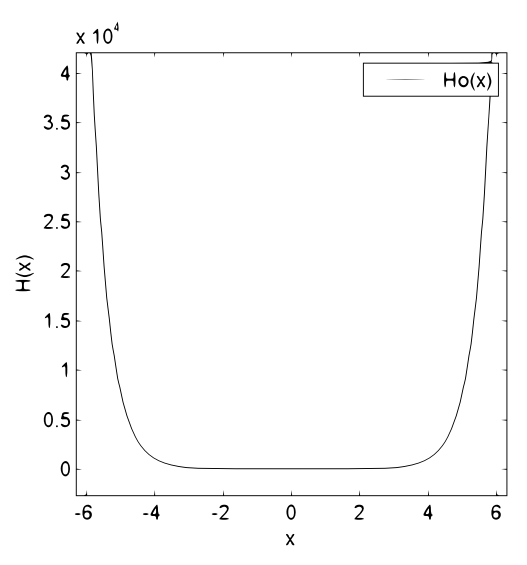

Figure 3 The solution of Eq.(4.3)

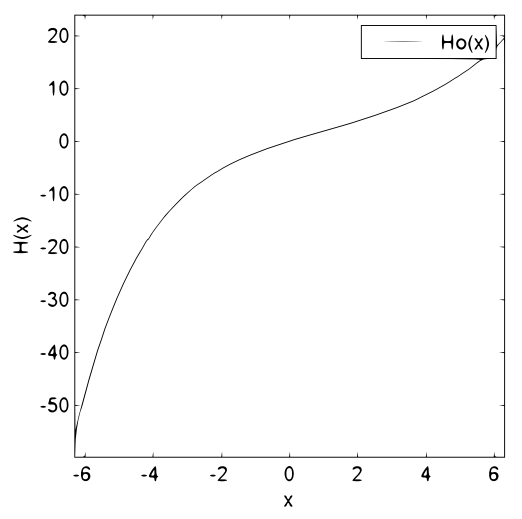

Example 4.3 Consider the following differential equation:

$$
\mathcal{H}^{\prime \prime}(x)+\frac{1}{6} \mathcal{H}^{\prime}(x)-\frac{1}{6} \mathcal{H}(x)=0, \quad \lim _{|x| \rightarrow \infty} \mathcal{H}(x)=0
$$

and the inequality

$$
\left|\mathcal{H}^{\prime \prime}(x)+\frac{1}{6} \mathcal{H}^{\prime}(x)-\frac{1}{6} \mathcal{H}(x)=0\right| \leq \epsilon E_{2}\left(x^{2}\right) \quad \forall x \in \mathbb{R}
$$

where $\mathcal{H} \in L^{\prime}(\mathbb{R})$.

Comparing with (3.10) and (3.11), we have, for $\alpha=2, a=\frac{1}{6}$ and $b=\frac{1}{6}$.

Using MATLAB, the solution of Eq. (4.3) is computed and depicted in Fig. 3.

By Theorem 3.4, problem (4.3) has a solution and is Hyers-Ulam-Mittag-Leffler stable with

$$
\begin{aligned}
\left|\mathcal{H}(x)-\mathcal{H}_{0}(x)\right| & \leq \frac{6 \epsilon}{5} \sum_{k=0}^{\infty} \frac{1}{\Gamma(2 k+1)} \int_{0}^{x} \mu^{2 k}\left(e^{\frac{-1}{2}(x-\mu)}-e^{\frac{1}{3}(x-\mu)}\right) d \mu \\
& \leq K \epsilon E_{2,2}\left(x^{2}\right),
\end{aligned}
$$

where $K=\frac{6}{5}$. 


\section{Conclusion}

This research has made an attempt to analyze the Mittag-Leffler-Hyers-Ulam and Mittag-Leffler-Hyers-Ulam-Rassias stability of linear differential equation with constant coefficients. Also we have showed that the Mittag-Leffler function and Fourier transform play an immodest role to prove the stability of differential equation. This new method of stability unifies different classes of differential equations, which may inspire further research in this domain.

\section{Acknowledgements}

Not applicable.

\section{Funding}

This work was supported by the Basic Science Research Program through the National Research Foundation of Korea funded by the Ministry of Education, Science, and Technology (NRF-2017R1D1A1B04032937).

\section{Availability of data and materials}

Not applicable.

\section{Competing interests}

The authors declare that they have no competing interests.

\section{Authors' contributions}

The authors equally conceived of the study, participated in its design and coordination, drafted the manuscript, participated in the sequence alignment, and read and approved the final manuscript.

\section{Author details}

${ }^{1}$ Department of Mathematics, Adhiyamaan College of Engineering, Hosur, Tamil Nadu, India. ${ }^{2}$ Research Institute for Natural Sciences, Hanyang University, Seoul 04763, Korea. ${ }^{3}$ Department of Mathematics, Government Arts and Science College, Hosur, Tamil Nadu, India. ${ }^{4}$ Department of Mathematics, Sri Vidya Mandir Arts and Science College, Uthangarai, Tamil Nadu, India.

\section{Publisher's Note}

Springer Nature remains neutral with regard to jurisdictional claims in published maps and institutional affiliations.

Received: 31 March 2020 Accepted: 23 July 2020 Published online: 31 July 2020

\section{References}

1. Momoniat, E., Myers, T.G., Banda, M., Charpin, J.: Differential equation with applications to industry. Int. J. Differ. Equ. 2012, Article ID 491874 (2014)

2. Ulam, S.M.: Problem in Modern Mathematics. Science Editors, Willey, New York (1960)

3. Hyers, D.H.: On the stability of linear functional equation. Proc. Natl. Acad. Sci. USA 27, $222-224$ (1924)

4. Rassia, T.M.: On the stability of the linear mapping in Banach spaces. Proc. Am. Math. Soc. 72, 297-300 (1978)

5. Alsina, C., Ger, R.: On some inequalities and stability results related to the exponential function. J. Inequal. Appl. 2, 373-380 (1998)

6. Razaei, H., Jung, S., Rassias, T.M.: Laplace transform and Hyers-Ulam stability of linear differential equation. J. Math. Anal. Appl. 403, 244-251 (2013)

7. Algifiary, Q.H., Jung, S.: Laplace transform and generalized Hyers-Ulam stability of linear differential equation. Electron. J. Differ. Equ. 2014, Article ID 80 (2014)

8. Wu, G., Baleanu, D.: Stability analysis of impulsive fractional difference equations. Fract. Calc. Appl. Anal. 21, 354-375 (2018)

9. Wu, G., Baleanu, D., Huang, L.: Novel Mittag-Leffler stability of linear fractional delay difference equations with impluse. Appl. Math. Lett. 82, 71-78 (2018)

10. Wu, G., Abdeljawad, T., Liu, J., Baleanu, D., Wu, K.: Mittag-Leffler stability analysis of fractional discrete-time neural networks via fixed point technique. Nonlinear Anal., Model. Control 24, 919-936 (2019)

11. Mittag-Leffler, G.M.: Sur la nouvelle fonction of $E_{\alpha}(x)$. C. R. Acad. Sci. Paris 137, 554-558 (1903)

12. Wiman, A.: Uber de fundamental Satz in der theorie der theorie der funktionen. Acta Math. 29, 191-201 (1905)

13. Agarwal, R.P.: A propos d'une note M. Pierre Humbert. C. R. Acad. Sci. Paris 236, 2031-2032 (1953)

14. Humbert, P., Agarwal, R.P.: Sur la fonction de Mittag-Leffler et quelquesunes de ses generalizations. Bull. Sci. Math. 77, 180-186 (1953)

15. Kilbas, A., Srivastava, H., Trujillo, J.: Theory and Applications of Fractional Differential Equation. North-Holland Mathematical Studies, Elsevier Science, Amsterdam (2006)

16. Osgood, B.G.: Lectures on the Fourier Transform and Its Application. Pure and Applied Undergraduate Texts, vol. 33. Am. Math. Soc., Providence (2009)

17. Folland, G.B.: Fourier Analysis and Its Applications. The Wadsworth \& Brooks/Cole Mathematical Series. Wadsworth \& Brooks/Cole Advanced Books \& Software, Pacific Grove (1992) 
18. Kalvandi, V., Eghbali, N., Rassias, J.M.: Mittag-Leffler-Hyers-Ulam stability of fractional differential equation of second order. J. Math. Ext. 13, 29-43 (2019)

19. Liu, K., Wang, J., O'Regan, D.: Ulam-Hyers-Mittag-Leffler stability for $\psi$-Hilfer fractional-order delay differential equations. Adv. Differ. Equ. 2019, Article ID 50 (2019)

20. Liu, K., Wang, J., Zhou, Y., O'Regan, D.: Ulam-Hyers stability and existence of solution for fractional differential equation with Mittag-Leffler kernel. Chaos Solitons Fractals 132, Article ID 109534 (2020)

21. Niazi, A.U.K., Vel̆, T., Rekhman, M.U., Denkhao, P.: Ulam-Hyers-Mittag-Leffler stability for nonlinear fractional-order neutral type differential equations. Sb. Math. 209, 1337-1350 (2018)

22. Abbas, M.l.: Existence and uniqueness of Mittag-Leffler-Ulam stable solution for fractional integro differential equation with nonlocal initial conditions. Eur. J. Pure Appl. Math. 8(4), 478-498 (2015)

23. Kalvandi, V:: Mittag-Leffler-Hyers-Ulam stability of fractional differential equation. Glob. Anal. Discrete Math. 3, 21-31 (2018)

24. Sontakke, B.R., Kamble, G.B., UI-Haque, M.M.: Some integral transform of generalized Mittag-Leffler functions. Int. J. Pure Appl. Math. 108(2), 327-339 (2016)

25. Wang, J.R., Zhou, Y.: Mittag-Leffler-Ulam stabilities of fractional evolution equations. Appl. Math. Lett. 25, 723-728 (2012)

26. Eghbai, N., Kalvandi, V., Rassias, J.M.: A fixed point approach to the Mittag-Leffler-Hyers-Ulam stability of a fractional integral equation. Open Math. 14, 237-246 (2016)

27. Wang, J.R., Zhang, Y.: Ulam-Hyers-Mittag-Leffler stability of fractional-order delay differential equation. Optimization 63, 1181-1190 (2014)

28. Moharramnia, A., Eghbali, N., Rassias, J.M.: Mittag-Leffler-Hyers-Ulam-Rassias stability of deterministic semilinear fractional Volterra integral equation and of stochastic systems by Brownian motion. UPB Sci. Bull., Ser. A 82, 103-110 (2020)

29. Deitmar, A.: A First Course in Harmonic Analysis. Springer, Germany (2005)

30. Katzenelson, Y.: An Introduction to Harmonic Analysis. Cambridge University Press, Cambridge (2002)

\section{Submit your manuscript to a SpringerOpen ${ }^{\circ}$ journal and benefit from:}

- Convenient online submission

- Rigorous peer review

Open access: articles freely available online

- High visibility within the field

- Retaining the copyright to your article

Submit your next manuscript at $\gg$ springeropen.com 